\title{
Cephalometric Approach to Vertical facial Height
}

\author{
Dr Suvit Maskey,' Dr Rosha Shrestha² \\ 'Asst.Profesor, Department of dentistry, Institute of Medicine,Kathmandu, Nepal \\ ${ }^{2}$ Asso. Professor, KIST Medical College and Teaching Hospital Lalitpur, Nepal
}

Correspondence: Dr Suvit Maskey; Email: docsuvit@gmail.com

\section{ABSTRACT}

Introduction: The vertical heights and the proportions are very important for balance of the face and attractiveness along with the good smile. Harmonious vertical proportions and the Antero-posterior relations contribute to aesthetics. Therefore it is necessary to determine the vertical facial height and the ratios in treatment planning of the patients. The aims and objectives of this study are to compare the vertical facial heights of patients considering the Lower Anterior Facial Height (LAFH), the Upper Anterior Facial Height (UAFH), Total Anterior Facial Height (TAFH) and Posterior Facial Height (PFH) among the sexes in Class I skeletal group obtained from cephalometric analysis.

Materials \& Method: The study material comprised of 52 patients (24 males and 28 females) and lateral cephalometric radiographs were traced with Skeletal Class I relationship. Cephalometric analysis was performed by using hand tracing. Independent t tests were used for comparisons.

Result: The total anterior facial height (TAFH), lower anterior facial height (LAFH), upper anterior facial height (UAFH), total posterior facial height (TPFH) measurements showed no significant differences between both sexes in Skeletal Class I group but statistically significant difference in ratios of posterior facial height and total anterior facial height and also there was statistically significant differences in the ratio between upper facial height and the total anterior facial height ( $p$ value <0.05).

Conclusion: This cephalometric analysis can be applied to determine the vertical height of occlusion which can be helpful in prosthetic rehabilitation of edentulous patients.

Keywords: Cephalometry, Sex Variation, Vertical Facial Height

\section{INTRODUCTION}

There is always a desire to improve with the demand of a patient who come to us with a problem, to have better facial expression, harmony of the face, better smile as well as functions. Here the vertical pattern and the proportions has a major role in harmony of the face and its attractiveness along with the smile to give the beauty ${ }^{1,2}$ a well balanced face always includes a harmonious vertical proportions and the Anteroposterior relations of the jaws. A good knowledge of optimal facial proportions in their population can be very helpful in the treatment planning to improve aesthetic as well as to regain functions. ${ }^{3}$

Vertical dimensions included Anterior Facial Height (AFH) and Posterior Facial Height (PFH). In cephalometry anterior Facial Height (AFH) is divided in to upper anterior facial height (UAFH) and lower anterior facial height (LAFH). Determination of the vertical occlusion dimension is a very important, in order to regain the proper function, physiology as well as the aesthetics ${ }^{4,5}$ for the patients with the tooth loss.

Tooth loss and the loss of support to the soft tissue provided by the natural dentition cause the alteration of vertical dimension including changes in hard and soft tissue of face and jaw region which leads to drastic changes in the vertical dimension of occlusion

The determination of correct vertical dimension of occlusion has always been of great importance as well as challenge and also for the fabrication of prosthodontic rehabilitation ${ }^{5,6}$

Any errors in the vertical dimension can affect the stomatognathic system ${ }^{7}$ leading to temporomandibular joint disorder, excessive bone resorption, muscle dysfunction, altered phonetics and inappreciable aesthetics with difficulty in mastication and deglutition. Whereas reconstruction of original occlusion and Vertical Occlusal dimension by a proper prosthesis leads to a better adaptation, recovery of the functions with satisfaction. 
The aim of this research was to determine vertical Dimension of Occlusion for predictable occlusal rehabilitation and to compare the Anterior Facial Height (AFH) and Posterior Facial Height (PFH) in both sexes.

\section{MATERIALS AND METHOD}

The study was performed in 52 Lateral Cephalograms collected from KIST Medical college and teaching hospital where 24 were male and 28 were females. The age range was between 25 - 35 years. The inclusion criteria were skeletal class I jaw relationship $\left(A N B=2^{\circ}-4^{\circ}\right)$, facial and jaw symmetry, no history of previous orthodontic, Prosthodontic treatment or any surgical reconstruction or maxillofacial surgery. The Cephalograms were traced manually on acetate paper using an X-Ray viewer.To assess the error, 20 lateral Cephalograms were randomly selected then re tracing was done followed by the measurements recheck. The linear measurements were recorded with the help of $\mathrm{mm}$ ruler and angular measurements with the help of protractor.

The following anatomical points were determined in the Cephalograms (FIGURE-1)

1. Nasion $(\mathrm{N})$ - the most anterior point of the naso frontal suture in the midsagittal plane,

2. Sella $(S)$ the middle of the sella turcica contour in the medial plane

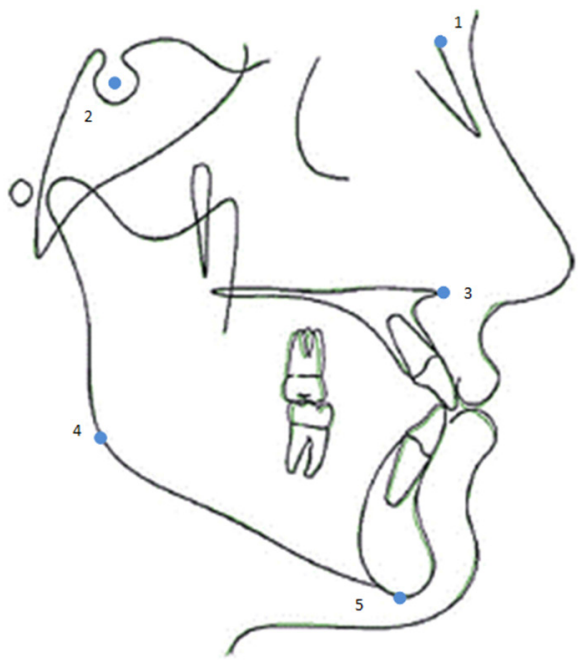
1. $\operatorname{Nasion}(\mathrm{N})$
4. Gonion (Go)
2. Sella (S)
5. Menton (Me)
3. Anterior Nasal Spine (ANS)

3. Anterior Nasal Spine (ANS) - tip of the anterior nasal spine

4. Gonion (Go) - the point at the intersection of the tangent of the lower border of mandible and the ramal plane

5. Menton (Me) - the lowest point on the lower border of mandibular symphysis

The Following linear skeletal cephalometric parameters were examined (Figure - 2)

1. Total Anterior Facial height (TAFH): Nasion (N) to Menton (Me)

Linear distance in millimeters from point Nasion (N) and point Menton (Me) [8]

2. Total Posterior Facial height (TPFH): Sella (S) to Gonion (Go)

Linear distance in millimeters from point Sella (S) to point Gonion (Go)

3. Upper Anterior Facial Height (UAFH): Nasion (N) to Anterior Nasal Spine (ANS) [9]

Linear distance in millimeters from point Nasion $(\mathrm{N})$ to point Anterior Nasal Spine (ANS)

4. Lower Anterior Facial height (LAFH): Anterior Nasal Spine (ANS) to Menton (Me) [9]

Linear distance in millimeters from point Anterior Nasal Spine (ANS) to point Menton (Me)

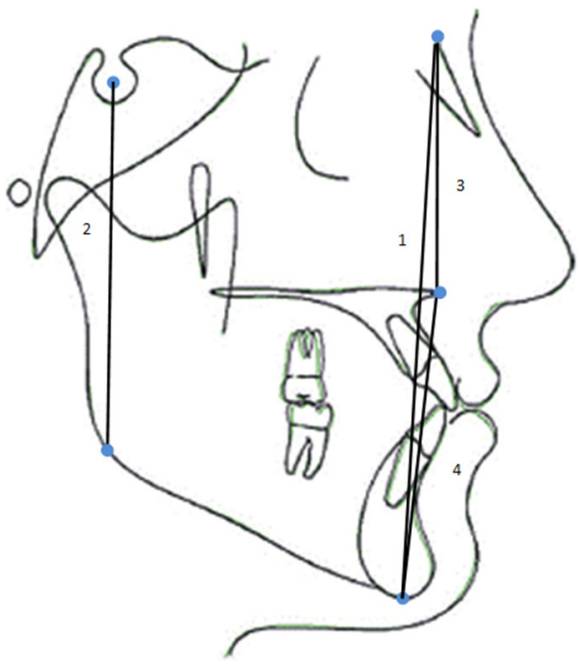

1. Total Anterior Facial height (TAFH)

2. Total Posterior Facial height (TPFH)

3. Upper Anterior Facial Height (UAFH)

4. Lower Anterior Facial height (LAFH)

Figure 2: Cephalometric measurements 


\section{RESULT}

Sagittal Skeletal jaw relationship was determined by using ANB value between $2^{\circ}-4^{\circ}$. The minimum value of SNA angle is $71^{\circ}$ and maximum is $93^{\circ}$ with mean and standard deviation $82.4 \pm 5.28$ whereas the minimum value of SNB angle is $68^{\circ}$ and maximum is $91^{\circ}$ with mean and standard deviation $79.93 \pm 5$.40.The range of ANB angle is $2^{\circ}-4^{\circ}$ with mean and standard deviation 2.60 \pm 0.81 (Table 1)

The mean value of posterior facial height was 72.23 with standard deviation 0.70 whereas the mean value of the anterior facial height was 51.78 with standard deviation 0.71.Similarly the mean and standard deviation of the lower anterior facial height and the upper anterior facial height were $60.46 \pm 0.59$ and $51.78 \pm 0.79$ respectively. (Table 2 )

The mean value of Posterior Facial height (PFH) is greater in male than in female(male :75.16 \pm 0.79 , female: $69.71 \pm 0.49$ ). The mean value of Upper Anterior Facial height (UAFH) is greater in female than in male (male : $49.75 \pm 0.41$, female $53.53 \pm 0.98$ ). The mean value of Lower Anterior Facial height (LAFH) is almost similar in both sexes( male: 60.83 \pm 0.67 , female:60.14 $\pm 0.51)$. The mean value of Total Anterior Facial height

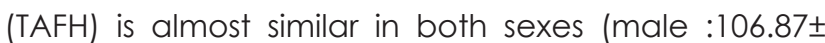
0.84 ,female:104.57 \pm 0.57$)$.Statistical analysis of data between sexes showed that there is no significant differences in Upper Anterior Facial height (UAFH), Lower Anterior Facial height (LAFH) and Total Anterior Facial height (TAFH) and linear dimensions (N-Me, $\mathrm{N}$-ANS, ANS-Me) but statistically significant difference in Posterior Facial height (PFH) between sexes (S-Go p value *0.04 ) (Table 3)

The mean ratio of Total Anterior Facial height (TAFH) and Posterior Facial height (PFH) in male is $1.42 \pm 0.11$ whereas in female is $1.50 \pm 0.13$. Students t test was performed and there was significant differences in the ratio between Posterior Facial height (PFH) and the Total Anterior Facial height (TAFH) between sexes ( $P$ value is *.027) .The mean ratio of Upper Anterior Facial height (UAFH) and Lower Anterior Facial Height (LAFH) in male is $0.82 \pm 0.086$ whereas in female is $0.90 \pm 0.20$.

The mean ratio of Upper Anterior Facial height (UAFH) and Total Anterior Facial height (TAFH) in male is $0.46 \pm 0.02$ whereas in female is $0.51 \pm 0.10$.There was statistically significant differences in the ratio between

Table 1: Showing the cephalometric analysis of the angular parameters $(\mathrm{N}=52)$.

\begin{tabular}{|l|c|c|c|c|c|}
\hline & Min & Max & Mean & S.D & 95\% \\
\hline SNA & 71.0 & 93.0 & 82.460 & 5.2888 & $80.987-83.932$ \\
\hline SNB & 68.00 & 91.00 & 79.9365 & 5.40613 \\
\hline ANB & 2.0 & 4.0 & 2.604 & .8177 \\
\hline
\end{tabular}

Table 2: Showing cephalometric analysis of the linear parameters of posterior facial height, upper anterior facial height, lower anterior facial height and total anterior facial height.

\begin{tabular}{|l|c|c|c|c|}
\hline & Mean & S.D & Min & Max \\
\hline S-GO & 72.231 & 0.70227 & 56.0 & 92.0 \\
\hline N-ANS & 51.788 & 0.79146 & 41.0 & 76.0 \\
\hline ANS-ME & 60.46 & 0.5929 & 45.0 & 75.0 \\
\hline N-ME & 105.635 & 0.71539 & 84.0 & 123.0 \\
\hline
\end{tabular}

Table 3 : Showing cephalometric analysis of the linear parameters in relation to gender.

\begin{tabular}{|l|c|c|c|c|c|c|}
\hline \multirow{2}{*}{ S-GO } & Gender & $\mathbf{N}$ & Mean & Std. Deviation & T value & P value \\
\hline \multirow{2}{*}{ N-ANS } & Male & 24 & 75.167 & 0.79873 & 3.002 & $* 0.04$ \\
\cline { 2 - 7 } & Female & 28 & 69.714 & 0.49580 & & -1.82 \\
\hline \multirow{2}{*}{ ANS-ME } & Male & 24 & 49.750 & 0.41729 & & 0.072 \\
\cline { 2 - 7 } & Female & 28 & 53.536 & 0.98300 & & 0.415 \\
\hline \multirow{2}{*}{ N-ME } & Male & 24 & 60.83 & 0.6799 & & 0.58 \\
\cline { 2 - 7 } & Female & 28 & 60.14 & 0.5176 & & 0.162 \\
\hline
\end{tabular}


Table 4: Showing cephalometric analysis of the Total Anterior Facial Height (TAFH) to Posterior Facial Height (PFH) in relation to gender.

\begin{tabular}{|c|c|c|c|c|c|c|}
\hline & Gender & $\mathbf{N}$ & Mean & Std. Deviation & T value & $P$ value \\
\hline \multirow{2}{*}{$\begin{array}{l}\text { Total Anterior Facial height (TAFH) vs } \\
\text { Posterior Facial height (PFH) }\end{array}$} & Male & 24 & 1.4294 & 0.11069 & \multirow{2}{*}{-2.274} & \multirow{2}{*}{$* 0.027$} \\
\hline & Female & 28 & 1.5071 & 0.13222 & & \\
\hline \multirow{2}{*}{$\begin{array}{l}\text { Upper Anterior Facial height (UAFH) vs } \\
\text { Lower Anterior Facial Height (LAFH) }\end{array}$} & Male & 24 & 0.8243 & 0.08676 & \multirow{2}{*}{-1.672} & \multirow{2}{*}{0.101} \\
\hline & Female & 28 & 0.9004 & 0.20752 & & \\
\hline \multirow{2}{*}{$\begin{array}{l}\text { Upper Anterior Facial height (UAFH) vs Total } \\
\text { Anterior Facial height (TAFH) }\end{array}$} & Male & 24 & 0.4662 & 0.02900 & \multirow{2}{*}{-2.374} & \multirow{2}{*}{$* 0.024$} \\
\hline & Female & 28 & 0.5137 & 0.10112 & & \\
\hline \multirow{2}{*}{$\begin{array}{l}\text { Lower Anterior Facial Height (LAFH) vs Total } \\
\text { Anterior Facial height (TAFH) }\end{array}$} & Male & 24 & 0.5683 & 0.03168 & \multirow{2}{*}{-0.767} & \multirow{2}{*}{0.447} \\
\hline & Female & 28 & 0.5745 & 0.02653 & & \\
\hline
\end{tabular}

Upper Anterior Facial height (UAFH) and the Total Anterior Facial height (TAFH) ( $\mathrm{P}$ value is less than * 0.024). The mean Lower Anterior Facial Height (LAFH) and Total Anterior Facial height (TAFH) ratio in male is $0.56 \pm 0.03$ whereas in female is $0.57 \pm 0.02$ (Table 4).

\section{DISCUSSION}

The present study found the facial height parameters in both sexes that is the Upper Anterior Facial height (UAFH) and Total Anterior Facial height (TAFH), the Lower Anterior Facial Height (LAFH) and the Total Anterior Facial height (TAFH) in Skeletal Class I group showed no significant differences. This agrees with the study done in Nigerian population by Obaidi et al and Yemitan Ta et $a^{10,11}$ and also there was no correlation found on Upper Anterior Facial height (UAFH) versus Lower Anterior Facial Height (LAFH) as the study done by Donald et al. ${ }^{12,10}$

This study also concludes that there is a statistically significant difference between Posterior Facial height (PFH) in between sexes. Posterior Facial height (PFH) is determined by lowering of the temporomandibular fossa and condylar growth. When condylar growth outpaces maxillary descent and eruption of the posterior teeth, forward rotation of the mandible occurs. Conversely, if dentoalveolar growth and growth of the maxillary sutures is greater than the growth at the condyles, backward rotation of the mandible is the result. Schudy et al Björk et al ans Solow et al ${ }^{13-15}$

This study also concludes that there is a significant difference in ratio of Posterior Facial height (PFH) and Total Anterior Facial height (TAFH) between males and females. This is also in agreement with the study done by Kharbanda et al. ${ }^{16}$

This study also determined that the Posterior Facial height (PFH) in male is $75.16 \pm 79$ whereas in females $69.7 \pm 49$, Upper Anterior Facial Height(UAFH) in male is $49.75 \pm 0.41$ whereas in female is $53.53 \pm 98$, Lower Anterior Facial Height(LAFH) in male is $60.83 \pm 0.67$ whereas in female is $60.14 \pm 0.51$ and the Total Anterior Facial height (TAFH) in male is $106.87 \pm 084$ and in female is $104.57 \pm 0.57$. (Table 3 ) . These values can be helpful in prosthodontic treatment of edentulous patients for rehabilitation of vertical dimension of occlusion along with other techniques.

\section{CONCLUSION}

Thus, the present study provides the clinician with a more specific reference in the vertical dimension of the face and the relationship of facial heights and its ratios in Skeletal Class I patients which would be helpful for rehabilitation of the missing natural dentition.

\section{OJN}




\section{REFERENCES}

1. Varlık SK, Demirbaş E, Orhan M. Influence of lower facial height changes on frontal facial attractiveness and perception of treatment need by lay people. The Angle Orthodontist 2010; 80; 6: 59-64.

2. Niaki EA, Arab S, Shamshiri A, Imani MM. The effect of the buccal corridor and tooth display on smile attractiveness. Australian Orthodontic Journal $2015 ; 31 ; 2: 195-200$.

3. Dent JP, The glossary of prosthodontic terms. Journal of Prosthetic Dentistry 2017; 117:1-105

4. Kato C, Fujita K, Kokai S. Increased occlusal vertical dimension induces cortical plasticity in the rat face primary motor cortex. Behavioural Brain Research 2012; 228; 2: 254-260.

5. Delic Z, Soskic MS, Grzic RP, et al. Evaluation of craniometric methods for determination of vertical dimension of occlusion. Coll Antropol 2000;1 : 31-35.

6. Strajnic L, Sinobad DS, Marcovic D, et al. Cephalometric indicators of the vertical dimension of occlusion. Coll Antropol 2008; 2: 535-541.

7. Chander N.G, Venkat R. An appraisal on increasing the occlusal vertical dimension in full occlusal rehabilitation and its outcome. Journal of Indian Prosthodontist Society2011; 11;2: 77-81

8. Jarabak JR, Fitzell JA. Light wire Edgewise appliance, Band I, Cephalometrics. Mosby Co, St Louis 1977; 128-166

9. Hasunda A, Boe OE, Jenatschke F, Nordeval K, Thunoldk DK, Wisth PJ. Clinical cephalometry for the Bergen-Technique. Orthodontic Department, University of Bergen 1977.

10. Obaidi H A. Variation of facial heights among the Class I, II and III dentoskeletal relationships (Cephalometric study). Al-Rafidain Dent J 2006; 4: 98-105.

11. Yemitan TA \& Oludarev YS. Vertical Facial Height and its Correlation with Skeletal Pattern among Young Nigerian Orthodontic Patients. International Journal of Dentistry and Oral Science 2018; 5: 661-666.

12. Donald G. Woodside, Sten Linder-Aronson. The channelization of upper and lower anterior face heights compared to population standard in males between ages 6 to 20 years. European Journal of Orthodontics 1979; 1; 1: 25-40.

13. Schudy FF. Vertical growth versus anteroposterior growth as related to function and treatment. Angle Orthod 1964; 34 : 75-93.

14. Björk A. Variations in the growth pattern of the human mandible: Longitudinal cephalometric study by the implant method. J Dent Res 1963; 400-411.

15. Solow B. The dentoalveolar compensatory mechanism: background and clinical implications. Br J Orthod 1980; 7: 145-161.

16. Kharbanda OP, Sidhu SS, Sundrum KR. Vertical proportions of face: a Cephalometric study. Int J Orthod 1991; Fall-Winter; 29 : 6-8. 